\title{
A New Sandwich ELISA Test Simultaneously Detecting E7 Proteins of HPV-16, 18 and 45 in Cervical Smears
}

Christina Metzger ${ }^{1 \#}$, Annabella Pittl ${ }^{1 \#}$, Andreas M. Kaufmann², Theodoros Agorastos ${ }^{3}$, Kimon Chatzistamatiou ${ }^{3}$, Oliver Böcher ${ }^{4}$, Werner Zwerschke ${ }^{1}$, Haymo Pircher ${ }^{1}$, Isabel Koch ${ }^{4}$, and Pidder Jansen-Dürr ${ }^{1 \S^{*}}$

${ }^{1}$ Institute for Biomedical Aging Research, Universität Innsbruck, Innsbruck, Austria

${ }^{2}$ Clinic for Gynecology, CBF, Charite-Universitätsmedizin Berlin, Berlin, Germany

${ }^{3} 4$ th Department of Obstetrics and Gynecology, Aristotle University of Thessaloniki, Greece

${ }^{4}$ Mikrogen $\mathrm{GmbH}$, Neuried, Germany

\#These authors contributed equally to the work

$\S$ Equally contributing senior authors

"Corresponding author: Dr. Pidder Jansen-Dürr, Institute for Biomedical Aging Research, Universität Innsbruck, Rennweg 10 , 6020 Innsbruck, Austria, Tel: 0043-512-58391944; Fax: 0043-512-5839198; E-mail: pidder.jansen-duerr@uibk.ac.at

Received date: Jul 26, 2016; Accepted date: Sep 14, 2016; Published date: Sep 21, 2016

Copyright: (C) 2016 Metzger C, et al. This is an open-access article distributed under the terms of the Creative Commons Attribution License, which permits unrestricted use, distribution, and reproduction in any medium, provided the original author and source are credited.

\begin{abstract}
Persistent infections by high-risk papilloma viruses (HPV) are the main etiological factor for cervical cancer, and E7 oncoproteins were suggested as new markers for tumor progression. The objective of this study was to generate a new Enzyme-Linked Immunosorbent Assay (ELISA)-based detection system to monitor expression of the E7 proteins of the high-risk HPV (hrHPV) types HPV-16, HPV-18, and HPV-45 in cervical smears. Using a combination of rabbit monoclonal antibodies raised against E7 proteins of HPV-16 and HPV-18/HPV-45, respectively, a trivalent E7-ELISA was developed and validated, using recombinant E7 proteins of various HPV types and lysates from E7positive cervical carcinoma cells. The amount of 0.5 picogram of E7 protein per well was determined as detection limit.

The E7-ELISA was used to determine E7 protein levels in cervical smears obtained from a total of 67 women. E7 protein concentration was below the detection limit in all HPV-negative smears, and E7 protein concentrations above background were found in some HPV-positive cervical samples. Together the work described herein provides a new tool for the simultaneous detection of E7 proteins of the three most prevalent in cervical neoplasia hrHPV types.
\end{abstract}

Keywords: Human papillomaviruses; E7 Oncoprotein; Cervical cancer screening

Abbreviations: CIN: Cervical Intraepithelial Neoplasia; ELISA: Enzyme-Linked Immunosorbent Assay; hrHPV: High-risk Human Papillomavirus; HERMES: HEllenic Real-life Multicentric Cervical Screening; PIPAVIR: Persistent Infections by Human PapillomaViruses.

\section{Introduction}

Persistent infections by human papillomaviruses (HPV) are the main etiologic factor for cervical pre-cancer and cancer [1-3]. About forty HPV genotypes can infect epithelial squamous and glandular cells in the cervical mucosa. Based on epidemiological and biochemical data only a subgroup of HPV types, referred to as high-risk HPVs, is associated with high-grade intraepithelial lesions that have a high potential for progression to invasive carcinoma. Infections by high-risk HPV genotypes have been detected in virtually all cervical cancers [1]. At least 15 high-risk HPV types have been associated with this cancer. HPV-16, HPV-18, and HPV-45 are the most prevalent genotypes in cervical cancer [4], collectively accounting for approximately $75 \%$ of all cervical carcinoma worldwide [5]. After infection by high-risk HPV types the control of viral gene expression is tightly regulated but becomes deregulated during progression to more severe dysplasia [3].
This leads to deregulated transcription of the early viral genes E6 and E7, and to cell proliferation and genomic instability[6], often resulting in the integration of episomal HPV genomes into host chromosomes $[7,8]$. This results in increased expression of the viral genes E6 and E7 [9], and an increase of E7 protein levels during early carcinogenesis [10]. The consistent overexpression of these two oncogenes is necessary and sufficient to induce and maintain the transformed phenotype of cervical cancer cells [11-13]. Since immortalization of keratinocytes by the E7 oncoprotein involves its ability to bind and thereby functionally inactivate cell-cycle regulatory proteins such as the retinoblastoma tumor suppressor protein $[10,14]$, the levels of E7 oncoproteins of carcinogenic HPV types may be specific markers for the detection of cervical pre-cancer and cancer.

Cytological assessment of cervical smears, by the Papanicolaou test $[15,16]$, suffers from a high rate of false negative results, which might be improved by the introduction of molecular biomarkers. New technologies for cervical cancer screening are urgently needed. Previous studies suggest that high-risk HPV E7 proteins are regularly expressed in cervical carcinoma and in their high-grade precursor lesions, suggesting that high-risk E7 oncoproteins are necessary for this cancer and may serve as new tumor biomarkers [10,17-20]. In the present study, we developed and validated a new ELISA test capable for the simultaneous detection of the E7 proteins of HPV-16, HPV-18, and HPV-45. 


\section{Material and Methods}

\section{Clinical samples}

Nineteen cervicovaginal samples tested positive for HPV 16 or 18 , and 48 samples tested negative for hrHPV were analysed for the present study (Table 1; extended clinical procedures are available in Supplementary File 1). 15 samples positive for HPV type $16(n=12)$ or $18(\mathrm{n}=3)$ came from a cohort of women aged 25 to 55 years who participated in the "Hellenic Real-life Multicentric Cervical Screening" (HERMES) study [21]. Four samples positive for HPV type $16(n=3)$ or $18(\mathrm{n}=1)$, as well as the $48 \mathrm{HPV}$ negative samples, came from the PIPAVIR (Persistent Infections with human Papilloma Viruses; www.pipavir.com) pre-study population. The classification of the cytology results was conducted according to the Bethesda 2001 directive [22]. All women samples were obtained in Thinprep PreservCyt Solution and stored at room temperature prior to E7 determination.

\section{Characterization of RabMAbs and polyclonal goat antibodies by direct ELISA}

E7 proteins of HPV types $11,16,18$ and 45 were purified as described [23]. The generation of rabbit monoclonal antibodies (RabMAbs) 42-3 and 143-7, as well as polyclonal goat antisera to HPV-16 E7 (Goat 1) and HPV-18 E7 (Goat 2), was described previously $[17,20]$. Increasing amounts of the E7 proteins encoded by HPV-16, HPV-18, HPV-45 and HPV-11 (low risk) were added in 100 $\mu \mathrm{l}$ coating buffer $\left(0.1 \mathrm{M} \mathrm{NaHCO}_{3}, \mathrm{pH}\right.$ 9.6) to each well of a 96-well plate (Maxisorp F, Nunc, Vienna, Austria) and incubated overnight at $4^{\circ} \mathrm{C}$. Wells were washed three times with washing buffer $(0.05 \%$ Tween20/1x PBS, pH 7.4). Subsequently, $300 \mu$ l blocking buffer (Mikrogen, Neuried, Germany) were added and incubated for $2 \mathrm{~h}$ at room temperature. $100 \mu \mathrm{l}$ diluent buffer (Mikrogen, Neuried, Germany) containing increasing amounts of RabMAbs or goat polyclonal antibodies were added to each well, followed by an incubation for $1 \mathrm{~h}$ at room temperature. Wells were washed three times with washing buffer. $100 \mu \mathrm{l}$ polyclonal HRP-conjugated secondary antibody (swine anti-rabbit antibody $(0.3 \mu \mathrm{g} / \mu \mathrm{l}$; Dako) or rabbit antigoat antibody $(0.5 \mu \mathrm{g} / \mu \mathrm{l}$; Dako) ) was added to each well and incubated for $1 \mathrm{~h}$ at room temperature. Wells were washed six times with washing buffer. $100 \mu \mathrm{l}$ tetramethylbenzidine (TMB) detection reagent es(HS)TMB (Seramun, Heidesee, Germany) was added to each well. Incubation was carried out for $30 \mathrm{~min}$ in the dark at room temperature. After adding $100 \mu \mathrm{l}$ stop solution $\left(25 \% \mathrm{H}_{3} \mathrm{PO}_{4}\right)$ to each well, absorbance was measured at $450 \mathrm{~nm}$ using a multilabel plate reader (Victor X5, Perkin Elmer, Vienna, Austria).

\section{Preparation of lysates from $\mathrm{CxCa}$ cells and clinical samples}

HeLa, CaSki, SiHa and MS751 cells were purchased from ATCC and grown in D-MEM containing 10\% fetal calf serum (FCS). When the appropriate cell density ( $90 \%$ confluent) was reached, cell lawns were washed with PBS followed by the addition of $2 \mathrm{ml}$ Trypsin/EDTA solution. After $5 \mathrm{~min}$ at $37^{\circ} \mathrm{C}$, excess trypsin was blocked by the addition of D-MEM containing 10\% FCS, and the cell suspension was transferred to a $15 \mathrm{ml}$ Falcon tube. Cells were resuspended in PBS and collected by centrifugation for $5 \mathrm{~min}$ at $224 \mathrm{~g}$. Subsequently, cells were resuspended in Thinprep PreservCyt Solution (Hologic, Crawley, UK) in a final concentration of 500,000 cells $/ \mathrm{ml}$. Cells were kept at room temperature for at least $24 \mathrm{~h}$. After homogenizing lysates for $30 \mathrm{~min}$ on an orbital shaker, an appropriate amount of lysate was centrifuged (10 min, $1,500 \mathrm{~g}, 4^{\circ} \mathrm{C}$ ). The pellet was resuspended in $150 \mu \mathrm{l}$ lysis buffer $\mathrm{A}$ (Mikrogen GmbH, Neuried, Germany) and centrifuged (30 s, 20,000 g, RT). Following the centrifugation step, $150 \mu \mathrm{l}$ lysis buffer B (Mikrogen $\mathrm{GmbH}$, Neuried, Germany) were added followed by vortexing for 1 min, which led to cell lysis. After centrifugation $\left(5 \mathrm{~min}, 20,000 \mathrm{~g}, 4^{\circ} \mathrm{C}\right)$, E7 protein concentration in supernatants was determined by sandwich ELISA.

Clinical samples stored at room temperature in Thinprep PreservCyt Solution were homogenized for $30 \mathrm{~min}$. Subsequently $40 \%$ of the homogenized suspension was centrifuged in $15 \mathrm{ml}$ Falcon tubes (10 min, $\left.1,500 \mathrm{~g}, 4^{\circ} \mathrm{C}\right)$. After discarding the supernatant, pellets were resuspended in $150 \mu \mathrm{l}$ lysis buffer $\mathrm{A}$. The suspension was centrifuged (30 s, 20,000 g, RT) and mixed with $150 \mu \mathrm{l}$ lysis buffer B. After a final centrifugation $\left(5 \mathrm{~min}, 20,000 \mathrm{~g}, 4^{\circ} \mathrm{C}\right.$ ), the supernatant was used for the detection of E7 protein using sandwich ELISA.

\section{Detection of HPV-16, 18 and 45 E7 by Sandwich ELISA}

96 well plates (Maxisorp F, Nunc, Vienna, Austria) were coated with $100 \mu$ coating buffer $\left(0.1 \mathrm{M} \mathrm{NaHCO}_{3}, \mathrm{pH}\right.$ 9.6) containing $0.25 \mu \mathrm{g}$ RabMAb 42-3 and 0.25 $\mu$ g RabMAb 143-7 and incubated overnight at $4^{\circ} \mathrm{C}$. After washing the wells three times with washing buffer, $300 \mu \mathrm{l}$ of blocking buffer were added to each well and incubated for $2 \mathrm{~h}$ at room temperature. Wells were washed three times with washing buffer followed by the application of recombinant protein, cell lysates or lysate from clinical samples, as appropriate. Subsequently wells were incubated for $1 \mathrm{~h}$ at room temperature. For detection of E7, a 1:1 mixture of biotinylated goat 1 and goat 2 antibodies diluted in diluent buffer was added to each well and incubated for $1 \mathrm{~h}$ at room temperature. Subsequently wells were washed 3 times with washing buffer and filled with $100 \mu \mathrm{l}$ streptavidin-poly-horseradish peroxidase (poly-HRP) conjugate (PolyHRP40; SDT, Baesweiler, Germany) diluted with diluent buffer. Following an incubation of $1 \mathrm{~h}$ at room temperature, wells were washed six times with washing buffer. $100 \mu \mathrm{l}$ of detection reagent were added to each well and incubated for $30 \mathrm{~min}$ in the dark at room temperature. The reaction was stopped by adding $100 \mu \mathrm{l}$ stop solution, and absorbance was measured at $450 \mathrm{~nm}$. E7 protein levels in cervical smears were calculated according to the standard curve obtained for recombinant E7 proteins.

\section{Results and Discussion}

\section{Characterization of anti-E7 antibodies}

To control the quality of antibodies selected for this study, direct ELISA experiments were performed. ELISA plates were coated with increasing concentrations of recombinant E7 proteins, and incubated with increasing amounts of purified RabMAbs 42-3 [17] and 143-7 [20], respectively. The bound rabbit monoclonal antibodies were detected by incubation with HRP-conjugated anti-rabbit-antibodies, incubated with substrate and quantitated by a Plate reader. Starting at an E7 protein concentration of 500 pg per well, OD values over the background could be obtained. For both RabMAbs, signal saturation was reached at a concentration of $50 \mathrm{ng}$ E7 protein per well and $81 \mathrm{ng}$ RabMAb (Figure 1A), indicating that the interaction between both RabMAbs and their cognate antigens is similar in quantitative terms (Figure 1A). We also tested the interaction of affinity-purified goat polyclonal antibodies raised against HPV-16 E7 (goat 1) and HPV-18 E7 (goat 2) with their cognate antigens. We found that signals over background were obtained with $10 \mathrm{ng}$ of HPV-16 E7 for goat 1 and 10 
Citation: $\quad$ Metzger C, Pittl A, Kaufmann AM, Agorastos T, Chatzistamatiou K, et al. (2016) A New Sandwich ELISA Test Simultaneously Detecting

Page 3 of 6

ng of either HPV-18 E7 or HPV-45 E7 with goat 2. Both goat antibodies displayed a weak but detectable cross reactivity with low- risk E7 proteins, as shown here for the E7 protein of HPV-11 (Figure 1B).

A
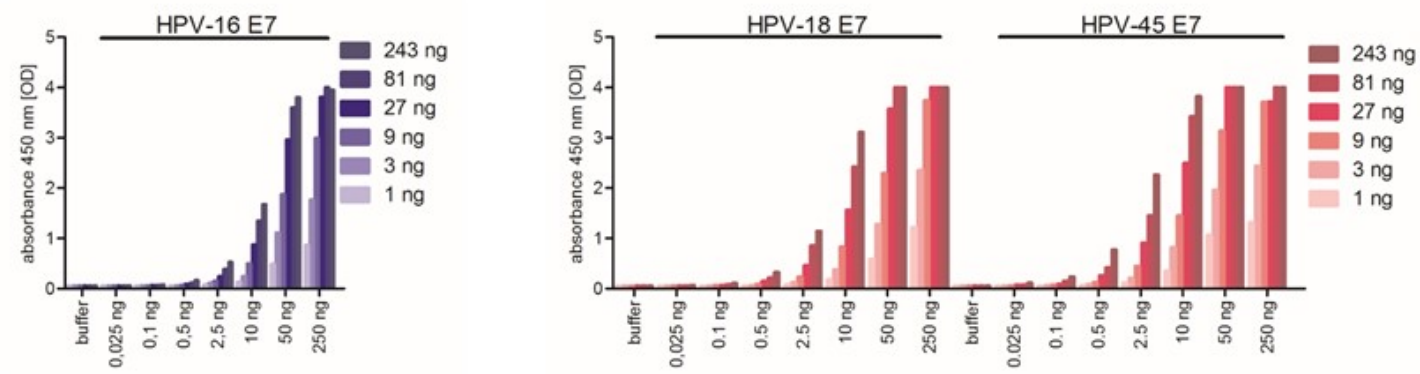

B
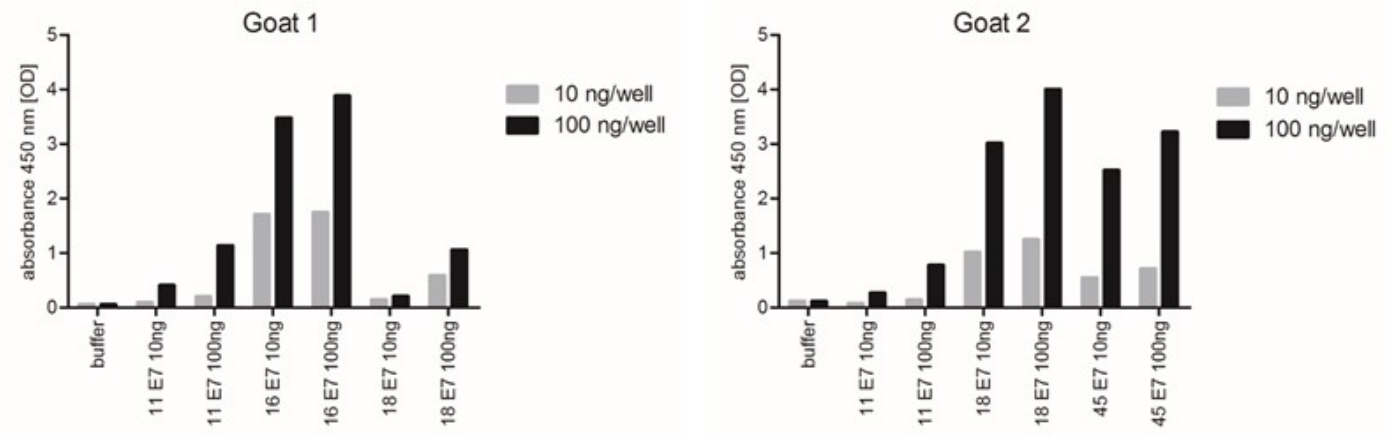

Figure 1: Characterization of E7 antibodies. ELISA plates were coated with increasing amounts (0.025-250 ng) of recombinant E7 proteins, as indicated, and incubated with (A) increasing amounts (1-243 ng) of a 1:1 mixture of RabMAbs 42-3 and 143-7, followed by a secondary polyclonal swine anti-rabbit antibody coupled to HRP, and (B) increasing amounts (10-100 ng) of antibodies Goat 1 and Goat 2, as indicated, followed by a secondary anti-goat antibody coupled to HRP. Absorbance at $450 \mathrm{~nm}$ was measured after incubation with TMB.

\section{Development of an E7 sandwich ELISA}

Based on the results of the direct ELISA assays, a sandwich ELISA was established by coating ELISA plates with a 1:1 mixture of RabMAbs 42-3 and 143-7. For detection of bound E7 proteins, a 1:1 mixture of biotinylated antisera goat 1 and goat 2 was used. This setting was then tested with an increasing concentration of the E7 proteins of HPV-16, HPV-18 and HPV-45. For HPV-16, HPV-18 and HPV-45, signal over background was obtained starting at a concentration of $500 \mathrm{fg}$ per well, and a steady increase of the signal was observed up to $10 \mathrm{pg}$ of each E7 protein (Figure 2A). In the same setting, the addition of $100 \mathrm{pg}$ of the low-risk E7 protein of HPV-11 gave no detectable signal. Multiple runs of the titration curves shown in Figure 2A were performed and used to determine the intra-assay and inter-assay variance of the ELISA (Table 1), which was found to be below $10 \%$ in all cases.

\begin{tabular}{|l|l|l|l|}
\hline & HPV-16 E7 & HPV-18 E7 & HPV-45 E7 \\
\hline Low range & $4 / 8$ & $3 / 7$ & $4 / 6$ \\
\hline Medium range & $5 / 9$ & $3 / 6$ & $3 / 6$ \\
\hline High range & $4 / 7$ & $6 / 7$ & $4 / 5$ \\
\hline
\end{tabular}

Table 1: Intra- and inter-assay variance (\%) of the E7 ELISA with recombinant E7 proteins of HPV types 16, 18 and 45.

To test the suitability of the sandwich ELISA for detection of endogenous E7 proteins present in cervical carcinoma cells, CaSki, SiHa (both HPV-16 positive), HeLa (HPV-18 positive) and MS-751 (HPV-45 positive) cells were grown and fixed in ThinPrep solution. The E7 protein content in extracts obtained from 20,000 and 50,000 cells each was determined by E7 ELISA, with signals over background being achieved with 20,000 cells per well in each case (Figure 2B). 
A
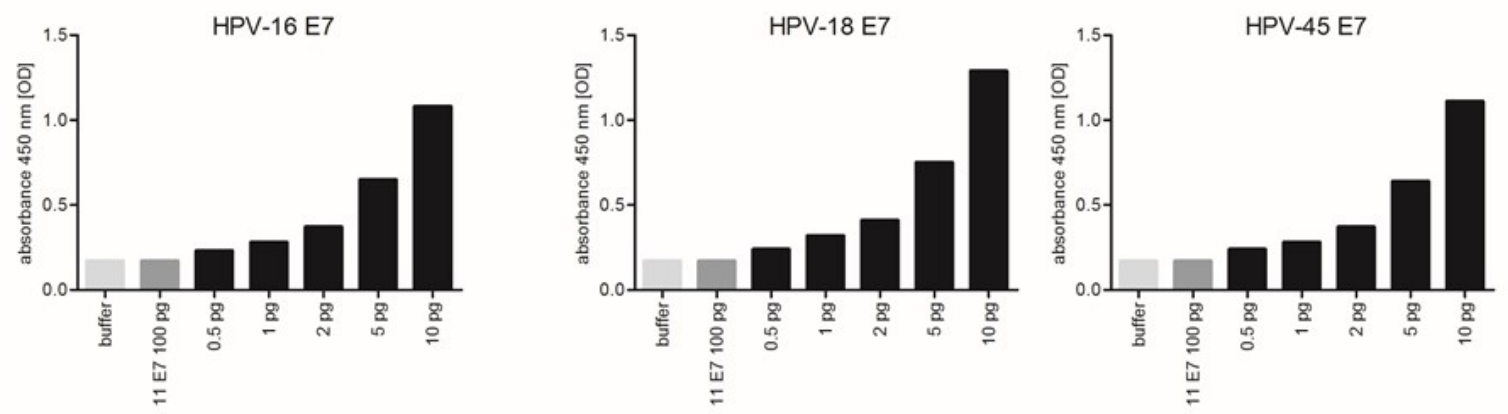

B
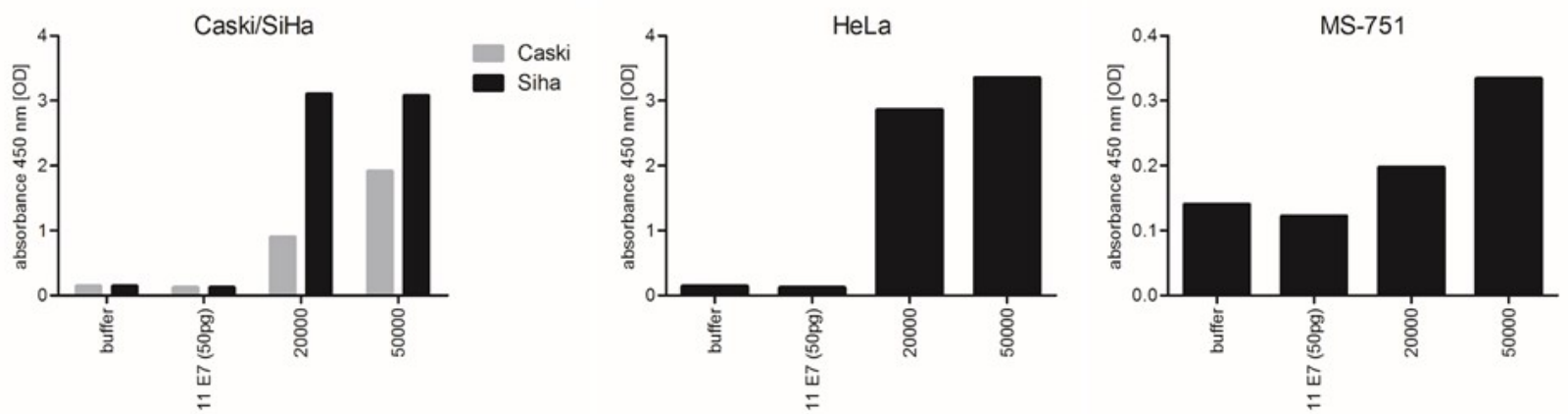

Figure 2: Setup and technical validation of a sandwich ELISA for the simultaneous detection of HPV-16, -18, -45 E7 proteins: ELISA plates were coated with a 1:1 mixture of monoclonal RabMAb 42-3 and 143-7. (A) Increasing amounts (0.5-100 pg) of recombinant HPV-E7 proteins were added as indicated, (B) protein extracts of CaSki, SiHa, HeLa, and MS751 cells prepared from 20,000 and 50,000 cells were added as indicated. Subsequently, a 1:1 mixture of biotinylated antibodies Goat 1 and Goat 2 was added, followed by the addition of SA-polyHRP40. After incubation with TMB, absorbance at $450 \mathrm{~nm}$ was measured. OD values were converted to E7 protein concentration according to the standard curve. HPV-11 E7 protein and lysis buffer were used as negative controls.

\section{Quantification of E7 proteins by sandwich ELISA in cervical smears}

For initial clinical assessment of the sandwich ELISA described here, a set of cervical smears from 67 women (obtained from the HERMES study and the PIPAVIR pre-study; Table S1) was analysed. Cervical smears from women were taken up in ThinPrep solution according to standard procedures and characterized by cytology. HPV DNA was determined by HPV DNA genotyping. We found that the set of clinical samples contained 15 smears which were positive for HPV-16 DNA and 4 smears positive for HPV-18 DNA, plus 48 smears in which no HPV DNA could be detected. Cellular material from ThinPrep samples was lysed and E7 concentration determined by E7 sandwich ELISA, based on the calibration curve obtained with recombinant E7 proteins before (Figure 2A). E7 protein expression was undetectable in all HPV-negative samples, indicating that endogenous proteins from patient tissue do not give rise to nonspecific background.
In the HPV-16 DNA positive samples and the HPV-18 DNA positive samples, concentration of the respective E7 concentration varied between 0 and $4 \mathrm{pg}$ per well (Figure 3). Unfortunately, the unavailability of HPV-45 positive samples precluded the validation of the test for HPV-45 E7 in clinical samples. Of note, the sandwich ELISA described here provides virtually no background signals for E7 proteins of HPV-16 in clinical samples. Background problems prevented previous attempts to establish a sensitive sandwich ELISA for HPV-16 E7 (our unpublished results). Probably, most available antibodies to HPV-16 E7 cross-react with epitopes present elsewhere in the human proteome. Reduction of background signals in this particular case is probably achieved by the fact that RabMab42-3, used here as coating antibody, recognizes in the $\mathrm{Zn}$ finger region of HPV-16 E7 protein a conformational epitope, which apparently is not represented in the cellular proteome [17]. 


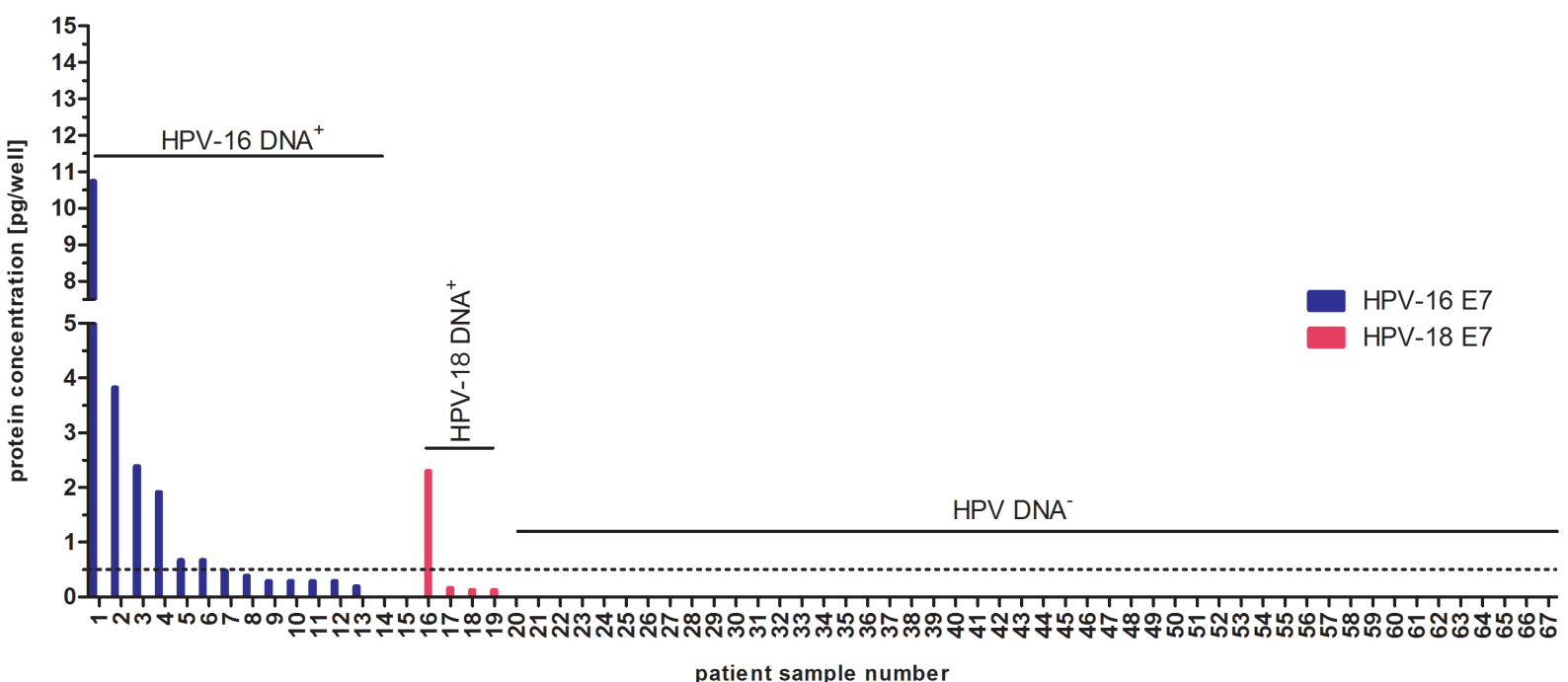

Figure 3: Detection of HPV-16 and -18 E7 in cervical smears: Lysates from HPV-16 and HPV-18 E7 positive cervical smear specimens as well as HPV E7 negative smears, as determined by DNA typing, were prepared and analyzed using the sandwich ELISA. Patients are numbered from 1 to 67, as indicated. Values shown in the figure are randomized to protein concentrations according to standard values determined for each single HPV E7 protein. The dotted line indicates the cut-off value of $500 \mathrm{fg}$ E7 protein per well.

Antibodies to E7 proteins of high-risk HPV types have been used previously to assess a potential role of these proteins in cervical cancer. On the one hand, sandwich ELISAs were described for the separate detection of the E7 proteins of HPV-16 and HPV-18, respectively, with a reported detection limit of one hundred picogram of E7 proteins [24]. Along the same lines, we have reported previously that $500 \mathrm{fg}$ of E7 protein of HPV-18 can be detected in a sandwich ELISA using goat serum 2 as coating antibody and biotinylated RabMab 147-3 as detection antibody [24]. On the other hand, several groups have used recombinant E7 proteins to set up ELISAs that allow detection of antibodies against E6 and E7 proteins of human papillomavirus types 16 and 18 in patient serum [25], thereby providing an alternative scenario to evaluate the role of E7 (and E6) proteins in cervical carcinogenesis.

The work described here establishes for the first time a sandwich ELISA suitable for the simultaneous detection of the E7 oncoproteins of HPV types 16, 18, and 45. Of note, reliable detection of HPV-16E7 in clinical samples was not achieved in previous studies using polyclonal goat serum (goat1) as coating antibody and biotinylated RabMab (RabMab 42-3) as detection antibody. This ELISA format revealed sporadic background signals which precluded its use with clinical samples (our unpublished data). In this communication, the problem was overcome by instead using a mixture of both RabMabs (42-3 and 143-7) as coating antibodies, and achieving detection with a mixture of affinity-purified and subsequently biotinylated polyclonal goat sera (goat1 and goat2). It is shown here that the current assay detects recombinant E7 proteins of these three HPV types with a detection limit of $500 \mathrm{fg}$ and has no reactivity towards E7 protein of the low risk HPV type 11 . The assay can be performed from cervical swabs stored in ThinPrep buffer, providing compatibility with current clinical procedures. Using this assay, we demonstrated that signals for E7 proteins were undetectable in smears derived from HPV DNA negative women, whereas E7 levels over background were obtained in some of the HPV DNA positive women.

The very low number of samples from patients with histologically confirmed cervical intraepithelial neoplasia is the main obstacle in our study, in order to verify the clinical utility of the E7 ELISA test, especially in the framework of triaging women with HPV DNA positive results or abnormal cytology. We provide however data showing that this new diagnostic molecular test is worthy to be evaluated in large studies with sufficient number of cases in the different subgroups, in order to determine its clinical performance.

\section{Conclusion}

A trivalent E7-ELISA was developed and validated, specifically detecting E7 proteins of HPV-16 and HPV-18/HPV-45, with a detection limit of 0.5 picogram. In cervical smears, E7 protein concentration was undetectable in all HPV-negative samples, and E7 protein concentrations above background were found in some HPVpositive samples. In summary, we provide here a new tool for the simultaneous detection of E7 proteins of the most prevalent in cervical neoplasia hrHPV types.

\section{Acknowledgement}

For expert technical support, we thank Ursula Schiller and Bianca Mandt.

\section{Authors' contributions}

HP, CM, AP, and IK developed and technically validated the trivalent E7 sandwich ELISA. CM, AP, and IK prepared lysates and tested them by E7-ELISA. AMK, TA, and KC provided clinical samples and information on their HPV status. IK, HP, WZ and PJ conceived 
the study and drafted the manuscript. All authors read and approved the final manuscript.

\section{Compliance with Ethical Standards}

\section{Funding}

Work in the laboratories of P.J., A.M.K, T.A., and O.B. was supported by the European Union through FP 7 project PIPAVIR (Project. No304927). P.J. also acknowledges funding by the County of Tyrol (Zervixkarzinom, Project. No WIF-273-01-00003/01-0150). The cobas HPV test kits and consumables for the HERMES study were provided by Roche Diagnostics (http://molecular.roche.com/).

\section{Conflict of interest}

I.K. and O.B. are employees of Mikrogen GmbH. P.J., H.P., and W.Z. are listed as inventors on a patent application relating to the E7 ELISA. $\mathrm{AMK}, \mathrm{TA}, \mathrm{CK}, \mathrm{AP}$, and $\mathrm{CM}$ declare that they have no conflict of interest.

\section{Ethical approval}

All procedures performed in studies involving human participants were in accordance with the ethical standards of the institutional and/or national research committee and with the 1964 Helsinki declaration and its later amendments or comparable ethical standards. Informed consent was obtained from all individual participants included in the study. This article does not contain any studies with animals performed by any of the authors.

\section{References}

1. Walboomers JM, Jacobs MV, Manos MM, Bosch FX, Kummer JA, et al. (1999) Human papillomavirus is a necessary cause of invasive cervical cancer worldwide. J Pathol 189: 12-19.

2. Castellsague X, Diaz M, de Sanjose S, Munoz N, Herrero R, et al. (2006) Worldwide human papillomavirus etiology of cervical adenocarcinoma and its cofactors: implications for screening and prevention. J Natl Cancer Inst 98: 303-315.

3. zur Hausen H (1999) Immortalization of human cells and their malignant conversion by high risk human papillomavirus genotypes. Semin Cancer Biol 9: 405-411.

4. Munoz N, Bosch FX, de Sanjose S, Herrero R, Castellsague X, et al. (2003) Epidemiologic classification of human papillomavirus types associated with cervical cancer. N Engl J Med 348: 518-527.

5. de Sanjose S, Quint WG, Alemany L, Geraets DT, Klaustermeier JE, et al. (2010) Human papillomavirus genotype attribution in invasive cervical cancer: a retrospective cross-sectional worldwide study. Lancet Oncol 11: 1048-1056.

6. McLaughlin-Drubin ME, Munger K (2009) The human papillomavirus E7 oncoprotein. Virology 384: 335-344.

7. Klaes R, Woerner SM, Ridder R, Wentzensen N, Duerst M, et al. (1999) Detection of high-risk cervical intraepithelial neoplasia and cervical cancer by amplification of transcripts derived from integrated papillomavirus oncogenes. Cancer Res 59: 6132-6136.

8. Schwarz E, Freese UK, Gissmann L, Mayer W, Roggenbuck B, et al. (1985) Structure and transcription of human papillomavirus sequences in cervical carcinoma cells. Nature 314: 111-114.
9. Romanczuk H, Howley PM (1992) Disruption of either the E1 or the E2 regulatory gene of human papillomavirus type 16 increases viral immortalization capacity. Proc Natl Acad Sci USA 89: 3159-3163.

10. Fiedler M, Muller-Holzner E, Viertler HP, Widschwendter A, Laich A, et al. (2004) High level HPV-16 E7 oncoprotein expression correlates with reduced pRb-levels in cervical biopsies. FASEB J 18: 1120-1122.

11. Munger K, Phelps WC, Bubb V, Howley PM, Schlegel R (1989) The E6 and E7 genes of the human papillomavirus type 16 together are necessary and sufficient for transformation of primary human keratinocytes. J Virol 63: 4417-4421.

12. Hudson JB, Bedell MA, McCance DJ, Laiminis LA (1990) Immortalization and altered differentiation of human keratinocytes in vitro by the E6 and E7 open reading frames of human papillomavirus type 18. J Virol 64: 519-526.

13. von Knebel Doeberitz M, Rittmuller C, Aengeneyndt F, Jansen-Durr P, Spitkovsky D (1994) Reversible repression of papillomavirus oncogene expression in cervical carcinoma cells: consequences for the phenotype and E6-p53 and E7-pRB interactions. J Virol 68: 2811-2821.

14. Dyson N, Howley PM, Munger K, Harlow E (1989) The human papilloma virus-16 E7 oncoprotein is able to bind to the retinoblastoma gene product. Science 243: 934-937.

15. Bray F, Ren JS, Masuyer E, Ferlay J (2013) Global estimates of cancer prevalence for 27 sites in the adult population in 2008. Int J Cancer 132: 1133-1145.

16. Ferlay J, Soerjomataram I, Dikshit R, Eser S, Mathers C, et al. (2015) Cancer incidence and mortality worldwide: sources, methods and major patterns in GLOBOCAN 2012. Int J Cancer 136: E359-386.

17. Dreier K, Scheiden R, Lener B, Ehehalt D, Pircher H, et al. (2011) Subcellular localization of the human papillomavirus 16 E7 oncoprotein in CaSki cells and its detection in cervical adenocarcinoma and adenocarcinoma in situ. Virology 409: 54-68.

18. Fiedler M, Ressler S, Campo-Fernandez B, Laich A, Jansen L, et al. (2005) Expression of the high-risk human papillomavirus type 18 and 45 E7 oncoproteins in cervical carcinoma biopsies. J Gen Virol 86: 3235-3241.

19. Ressler S, Scheiden R, Dreier K, Laich A, Muller-Holzner E, et al. (2007) High-risk human papillomavirus E7 oncoprotein detection in cervical squamous cell carcinoma. Clin Cancer Res 13:7067-7072

20. Ehehalt D, Lener B, Pircher H, Dreier K, Pfister H, et al. (2012) Detection of human papillomavirus type $18 \mathrm{E} 7$ oncoprotein in cervical smears: a feasibility study. J Clin Microbiol 50: 246-257.

21. Agorastos T, Chatzistamatiou K, Katsamagkas T, Koliopoulos G, Daponte A, et al. (2015) Primary screening for cervical cancer based on high-risk human papillomavirus (HPV) detection and HPV 16 and HPV 18 genotyping, in comparison to cytology. PLoS One 10: e0119755.

22. Solomon D, Davey D, Kurman R, Moriarty A, O'Connor D, et al. (2002) The 2001 Bethesda System: terminology for reporting results of cervical cytology. JAMA 287: 2114-2119.

23. Fiedler M, Campo-Fernandez B, Laich A, Moser B, Stockl P, et al. (2006) Purification and characterisation of the E7 oncoproteins of the high-risk human papillomavirus types 16 and 18. J Virol Methods 134: 30-35.

24. Selvey LA, Dunn LA, Murray B, Tindle RW, Frazer IH (1992) An ELISA capture assay for the E7 transforming proteins of HPV16 and HPV18. J Virol Methods 37: 119-127.

25. Sehr P, Zumbach K, Pawlita M (2001) A generic capture ELISA for recombinant proteins fused to glutathione S-transferase: validation for HPV serology. J Immunol Methods 253: 153-162. 